\title{
COMPARISON OF COMPONENTS OF FUSARIUM HEAD BLIGHT IN DOUBLED HAPLOID WHEAT LINES
}

\author{
M.G. CROMEY ${ }^{1}$, S.C. SHORTER ${ }^{1}$, W.B. GRIFFIN ${ }^{1}$, \\ C.A. MUNRO ${ }^{1}$ and D.R. LAUREN ${ }^{2}$ \\ ${ }^{1}$ Crop \& Food Research, Private Bag 4704, Christchurch \\ ${ }^{2}$ HortResearch, Private Bag 3123, Hamilton \\ Corresponding author: cromeym@crop.cri.nz
}

\begin{abstract}
Components of Fusarium head blight (FHB), caused by Fusarium spp., were examined in one Chinese and seven New Zealand wheat cultivars, and in 141 doubled haploid lines from a cross between a susceptible and a moderately resistant cultivar. Components measured were incidence of FHB, proportions of grains with visible FHB infection, mycotoxins levels in grain and proportion of grains infected with Fusarium. Lines were sown in a field trial that was inoculated with $F$. graminearum and mistirrigated to enhance infection. The Chinese cultivar, Nanjing, had the lowest level of FHB in ears and visibly infected grains and Fusarium infection in harvested grain. Two New Zealand cultivars also had low levels of FHB components, while two other cultivars had medium levels and three had high levels of infection. There were moderate to good correlations between most components of FHB, except that the proportions of visibly infected grains were poorly correlated with other components. Keywords: Fusarium graminearum, head scab, mycotoxins, host resistance.
\end{abstract}

\section{INTRODUCTION}

Fusarium head blight (FHB), which is caused by a complex of Fusarium spp., has become increasingly important in New Zealand wheat crops (Cromey et al. 2002). The species mixture has been shown to vary according to region, with $F$. graminearum often predominating in samples from the North Island (Sayer \& Lauren 1991; Cromey et al. 2001b), where the disease is most prevalent.

FHB is most easily recognised on immature ears where one or more spikelets in each ear become prematurely bleached. Sometimes large areas of ears may be affected. When infection is severe, pink or orange spore masses may occur on diseased spikelets. FHB can cause yield losses of $30-70 \%$ where conditions favour the disease, but, more importantly, grain from affected crops may be less palatable to livestock than healthy grain and may contain mycotoxins such as the trichothecenes deoxynivalenol (DON) and nivalenol (NIV) (Bai \& Shaner 1994). The quality of seed for sowing is also adversely affected through reduced rates of germination and emergence.

There are two stages of infection of wheat ears by Fusarium. Host plants are most susceptible to primary infection during anthesis when florets can be infected, especially during wet conditions (Bai \& Shaner 1994). Secondary spread between florets and spikelets occurs at later stages of crop development.

Wheat cultivars differ in resistance to FHB. It is generally considered that no cultivar is immune, a few are moderately resistant and most are susceptible (Parry et al. 1995). Given the moderate levels of resistance and lack of highly effective fungicides (Cromey et al. 2001a), effective management of FHB will probably rely on the integration of a number of control options (Dill-Macky \& Jones 2000), including disease resistance. 
New Zealand cultivars range from highly susceptible to moderately resistant to FHB (Cromey et al. 2002), and differences between cultivars in levels of Fusarium on harvested grain have also been recorded (Cromey et al. 2001b). Under New Zealand conditions, moderate levels of resistance to FHB are usually effective in preventing high levels of damage, except under conditions most highly conducive to the disease (Cromey et al. 2002).

Cultivar resistance to FHB can be assessed by measuring disease incidence, the proportion of visibly affected (tombstone) grains in harvested samples, Fusarium infection in grain, and mycotoxin levels in grain samples. While these measures may be correlated, it has been suggested that resistance to FHB and mycotoxins may be controlled by different genes (Bai \& Shaner 1994). Knowledge of how these measures may be related to each other in breeding lines will assist wheat breeders in selecting cultivars with appropriate levels, and types, of resistance to FHB.

This paper reports a series of inoculated field trials aimed at exploring the relationships between FHB measurements in cultivars and in doubled haploid progeny of a cross between a susceptible and a moderately resistant cultivar.

\section{MATERIALS AND METHODS}

One cultivar field trial (randomised complete block design with eight cultivars and four replicates) was sown at the Crop \& Food Research farm at Lincoln in 2001. Plot dimensions were six rows $(1.1 \mathrm{~m})$ by $1.5 \mathrm{~m}$. A field trial containing the cultivars Otane (highly susceptible to FHB) and Karamu (moderately resistant) and 141 doubled haploid progeny from F1s of a cross between Otane and Karamu (randomised complete block design with two replicates) was also sown in 2001. Plot dimensions were three rows $(0.6 \mathrm{~m}) \times 1.5 \mathrm{~m}$. A subset of 54 of these doubled haploids and the two parents (representing the range in FHB levels in 2001) were sown in 2002 in a randomised complete block design with two replicates. Plot dimensions were $0.6 \mathrm{~m} \times 1.5 \mathrm{~m}$.

Frozen suspensions of $F$. gramineaum spores were supplied by Karin Watson (Biological Culturing \& Research Laboratory, 1213 Kaipaki Rd, RD3, Cambridge). Because the exact time of anthesis varied between cultivars and doubled haploid lines, inoculations were carried out on three dates during the anthesis period of crop development. Each line was inoculated twice during anthesis. On the day of each inoculation, inoculum (approximately 1000 spores $/ \mathrm{ml}$ ) was applied in water using a knapsack sprayer at approximately 9 a.m. Mist irrigation was applied to plots for $10 \mathrm{~min}$ each hour using a Galcon E.Z. Model 2040 irrigation controller until 5 p.m. to maintain high humidity in plots and assist with infection. In addition, plots were misted automatically for $10 \mathrm{~min}$ every hour between $7 \mathrm{p} . \mathrm{m}$. and $8 \mathrm{a} . \mathrm{m}$. for a 2 -week period from the beginning of anthesis to aid disease development. Incidence of FHB was estimated by examining all ears in each plot. Plots were harvested when ripe. The proportion of visibly infected grains was estimated in a $50 \mathrm{~g}$ sample of harvested grain from each plot.

Grain samples were analysed for the presence of DON and NIV, the trichothecene mycotoxins commonly found in infected New Zealand wheat crops (Lauren et al. 1991). Samples of cv. Otane and Karamu and 16 doubled haploid lines were analysed in each year. Analysis was by a method adapted from that described by Lauren \& Ringrose (1997). The samples were extracted with acetonitrile:methanol:water (85:5:15), and aliquots subjected to clean-up through an alumina-carbon/cation exchange column, followed by hydrolysis to convert all trichothecenes to parent alcohols. After neutralisation, the extracts were passed through a 216 Trichothecene Charcoal-Column (Romer Labs, MO, USA). These refined extracts were then used for analysis by HPLC with UV detection at $245 \mathrm{~nm}$ and with confirmation at $254 \mathrm{~nm}$. Analysis for NIV and DON used a Zorbax SB-C8 column held at $35^{\circ} \mathrm{C}$ with a mobile phase of methanolwater (12:88). Under these conditions NIV in some samples was subject to interference, and in these instances NIV was analysed using a Zorbax SB-Phenyl column held at $35^{\circ} \mathrm{C}$ with a mobile phase of methanol-water (1:99). Concentrations of mycotoxins were expressed on a grain fresh weight (approximately 5-10\% moisture) basis. 
Infection by Fusarium spp. was measured in all cultivars in both years, in 16 doubled haploid lines in the first year, and in all doubled haploid lines in the second year. One hundred seeds per plot were surface-sterilised in $1 \%$ sodium hypochlorite for $10 \mathrm{~min}$, and plated on to potato dextrose agar (PDA) at 10 seeds per plate. Plates were incubated for 4 to 8 days on a laboratory bench and numbers of Fusarium colonies were counted. Species identification was carried out on PDA and on carnation leaf agar (CLA) using the methods of Burgess et al. (1994). Plates for species identification were incubated for 7 days in an alternating temperature regime: $25^{\circ} \mathrm{C}$ light $/ 20^{\circ} \mathrm{C}$ dark, with a $12 \mathrm{~h}$ photoperiod.

Data from the cultivar trial were statistically analysed with ANOVA. Relationships between variables in the doubled haploid trials were examined graphically, and by calculated correlation coefficients.

\section{Cultivar trial}

\section{RESULTS}

Fusarium head blight incidence (\% tillers infected) was highest on Otane and Kohika, very low on Monad, Pegasus II and Nanjing, and intermediate on Impact, Karamu and Torlesse (Table 1). Grain infection with Fusarium and percentage of visibly infected grains were low on Nanjing, Monad and Pegasus II, high on Otane, Kohika and Impact, and intermediate on Torlesse and Karamu. The ranking of cultivars for each measurement was similar, except for Impact, which had relatively high levels of visibly infected grains and Fusarium in grain compared with FHB incidence.

TABLE 1: Mean Fusarium head blight (FHB) incidence, and proportions of visibly infected grains and Fusarium in harvested grain in eight wheat (Triticum aestivum) cultivars in a field trial in 2001/02.

\begin{tabular}{lccc}
\hline & $\begin{array}{c}\text { \% ears with } \\
\text { FHB }\end{array}$ & $\begin{array}{c}\text { \% visibly infected } \\
\text { grains }\end{array}$ & $\begin{array}{c}\text { \% grains with } \\
\text { Fusarium }\end{array}$ \\
\hline Nanjing & 0.0 & 0.4 & 8.2 \\
Monad & 0.2 & 1.1 & 10.5 \\
Pegasus II & 2.2 & 1.2 & 11.8 \\
Torlesse & 9.0 & 2.1 & 34.2 \\
Karamu & 19.0 & 2.4 & 32.0 \\
Impact & 19.0 & 7.9 & 45.2 \\
Kohika & 30.8 & 7.4 & 39.8 \\
Otane & 51.0 & 8.3 & 48.0 \\
& & & \\
LSD $(\mathrm{P}=0.05, \mathrm{df}=21)$ & 11.3 & 2.3 & 14.0 \\
\hline
\end{tabular}

\section{Doubled haploids: comparisons between years}

There was much less disease in the 2002/03 growing season than in 2001/02, and therefore some of the moderately susceptible lines did not have visible FHB infection in $2002 / 03$. There was little rainfall during flowering and grain fill in $2002 / 03$, while there were reasonable levels in 2001/02.

The correlation between years for FHB incidence (Fig. 1a) was not particularly good $(\mathrm{r}=0.50)$, due to the relatively low FHB incidence in 2002/03 (mean of about $3 \%$ infected tillers) compared with 2001/02 (mean of about $12 \%$ infected tillers).

There was poor correlation between proportions of visibly infected grains (Fig. 1b) in the 2 years $(r=0.50)$, although lines with low counts in 2001/02 had low counts in $2002 / 03$. Lines with high levels of visibly infected grains in 2002/03 all had high levels in 2001/02. However, many of the lines with high counts in 2001/02 had low counts in $2002 / 03$, due to the overall low level of FHB in 2002/03. 
For the 12 lines tested in both years, there was a good correlation between years (Fig. 1c) for proportion of grains with Fusarium (r=0.75). The predominant Fusarium species isolated from harvested grains was $F$. graminearum ( $83 \%$ of isolations) which was the species inoculated on to plots, with lower levels of $F$. avenaceum (10\%) and F. culmorum $(7 \%)$.
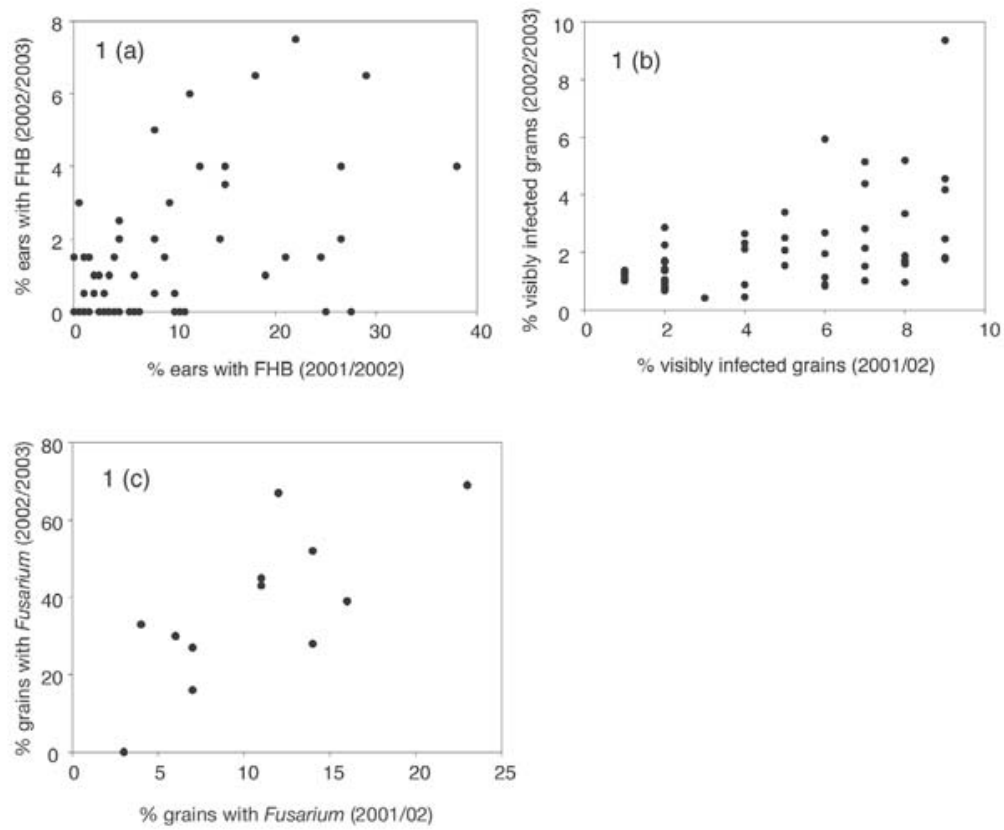

FIGURE 1: Relationships between years (2001/02, 2002/03) for Fusarium head blight (FHB) components in doubled haploid wheat (Triticum aestivum) lines from a cross between cv. Otane and cv. Karamu (a) FHB incidence, (b) proportion of visibly infected grains and (c) Fusarium levels in harvested grain.

\section{Doubled haploids: 2001/02 comparisons}

In 2001/2002, there was not a particularly good correlation ( $r=0.52)$ between percent FHB incidence and levels of visibly infected grains (Fig. 2a), except that all lines with higher levels of FHB also had high proportions of visibly infected grains. However, many of the lines with low proportions of FHB had high proportions of visibly infected grains.

There was a relationship between incidence of FHB and proportions of Fusarium in harvested grain (Fig. 2b) amongst the 16 lines that were tested for grain infection ( $r=0.77$ ). The cluster of lines with very low incidence of FHB included those lines with the lowest Fusarium incidence in harvested grain. The lines with the two highest FHB scores also had exceptionally high Fusarium incidences in harvested grain. There was a poor relationship amongst lines with intermediate (5-30\% FHB incidence), where the proportions of Fusarium infected grains ranged between 33 and $75 \%$.

The trichothecene DON was recorded in grain samples, while NIV was not detected. FHB incidence was compared with DON levels in harvested grain (Fig. 2c). Lines fell into two groups in the comparison ( $\mathrm{r}=0.77$ ). Lines with lower FHB incidence (up to 12\%) had a maximum of $1.3 \mathrm{mg} / \mathrm{kg}$ DON (mean $0.6 \mathrm{mg} / \mathrm{kg}$ ). Lines with higher FHB incidence had a 
range in DON of 1.1 to $4.3 \mathrm{mg} / \mathrm{kg}$ (mean $2.5 \mathrm{mg} / \mathrm{kg}$ ). There was a good correlation ( $\mathrm{r}=0.74$ ) between the proportions of grains with Fusarium and DON levels (Fig. 2d).
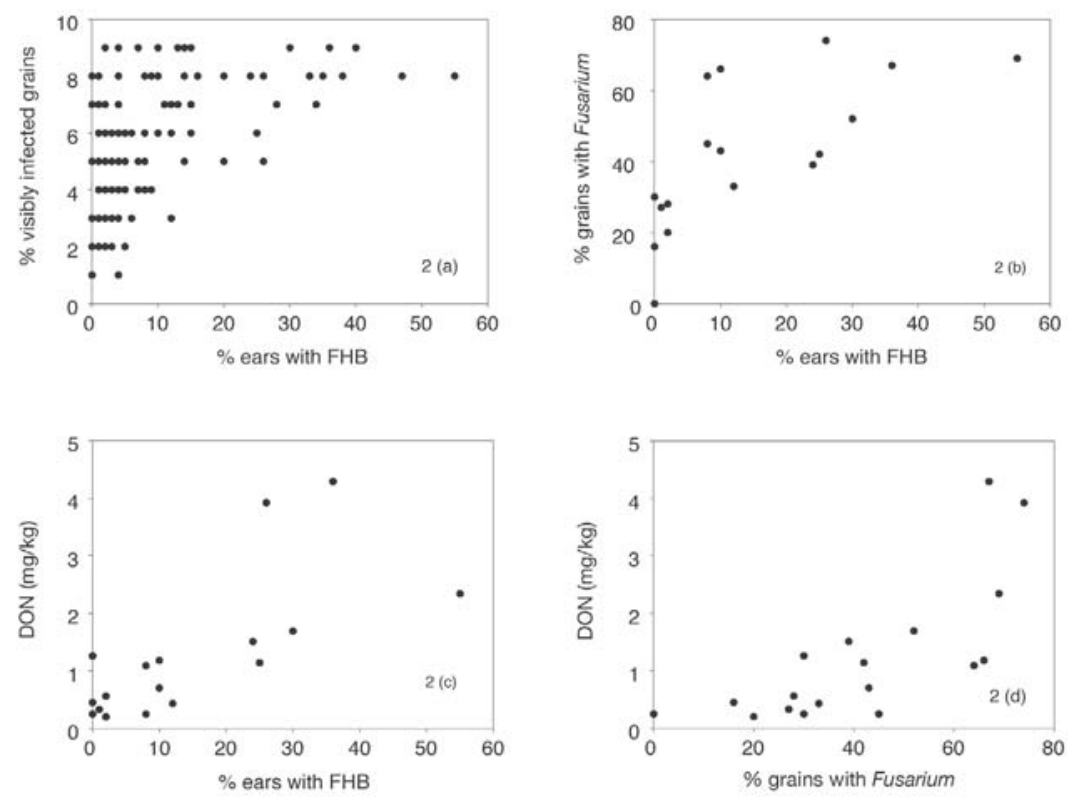

FIGURE 2: Relationships between Fusarium head blight (FHB) components in Karamu in an inoculated field trial in 2001/02 (a) FHB incidence and visibly infected grains, (b) FHB incidence and Fusarium levels in grain, (c) FHB incidence and levels of the mycotoxin DON and (d) F usarium levels in grain and levels of DON.

\section{Doubled haploids: 2002/03 comparisons}

There was no obvious correlation $(\mathrm{r}=0.41)$ between proportions of ears with FHB and the proportion of visibly infected grains in harvested samples (Fig. 3a). There was some correlation between proportions of ears with FHB and incidence of Fusarium infection in harvested grains $(\mathrm{r}=0.62)$, but there was virtually no correlation between these parameters at lower FHB levels (Fig. 3b). This probably reflects the low levels of visible FHB in 2002/03. Fusarium infection was relatively high in the five lines with greatest FHB incidence.

Both NIV and DON were recorded in grain samples from the 2002/03 trial, with average DON levels over 11 times greater than NIV. Results are presented as the total (NIV+DON) mycotoxin levels. The relationship between FHB incidence and mycotoxin levels (Fig. 3c) was good ( $\mathrm{r}=0.70$ ). However, two of the four lines with over $4 \%$ FHB had over $3.8 \mathrm{mg} / \mathrm{kg}$ NIV + DON, while the other two had only moderate mycotoxin levels. The four lines with intermediate FHB incidence had low to intermediate mycotoxin levels. There was a very good correlation $(\mathrm{r}=0.91)$ between incidence of Fusarium infection in grain and mycotoxin levels (Fig. 3d).

Two clusters of lines were evident (Fig. 3e) in the comparison between proportions of Fusarium infected grains and incidence of visibly infected grain $(\mathrm{r}=0.65)$. One group was low to moderate in Fusarium incidence (1\% to 22\%) and incidence of visibly infected 
grain $(0.2 \%$ to $3.8 \%)$. The other group was medium to high in Fusarium incidence (17\% to $37 \%)$ and in incidence of visibly infected grain $(2.3 \%$ to $9.5 \%)$, although there was no relationship between the 2 measurements within this group.
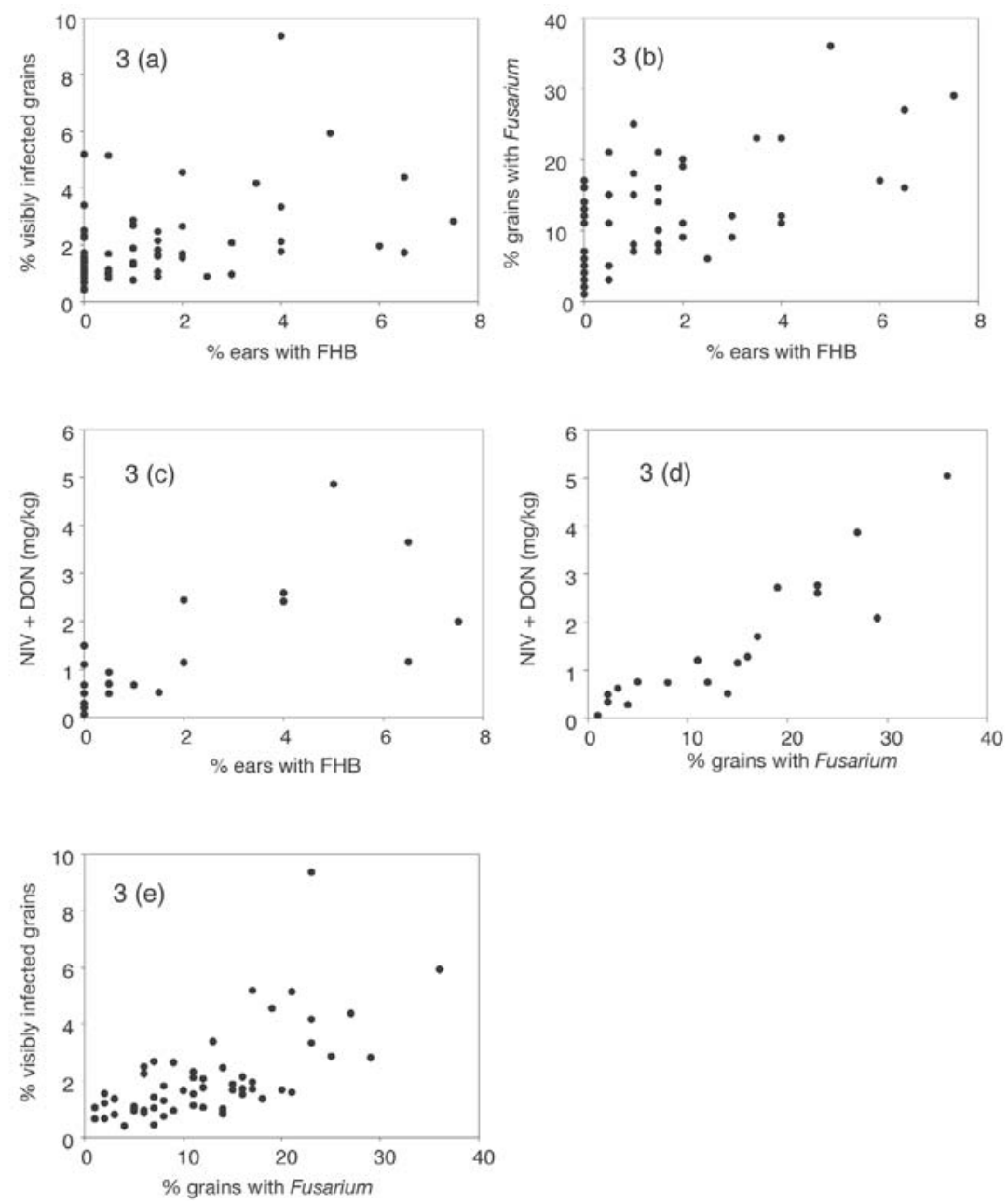

FIGURE 3: Relationships between Fusarium head blight (FHB) components in doubled haploid lines from a cross between wheat (Triticum aestivum) cv. Otane and cv. Karamu in a inoculated field trial in 2002/03 (a) FHB incidence and visibly infected grains, (b) FHB incidence and $F$ usarium levels in grain, (c) FHB incidence and levels of mycotoxins, (d) Fusarium levels in grain and mycotoxin levels and (e) F usarium levels in grain and visibly infected grains. 


\section{DISCUSSION}

Results of the cultivar trial confirm previous reports that New Zealand wheat cultivars differ in their susceptibility to FHB (Cromey et al. 2002). The New Zealand cultivars Otane and Kohika are confirmed to be highly susceptible to FHB. The Chinese cultivar, Nanjing, is reported to have a high level of resistance to FHB (Snijders 1990) and had the lowest level of FHB components in this trial. FHB components were also low in the New Zealand cultivars Monad and Pegasus II, which appear to have useful levels of resistance to the disease. The New Zealand cultivar Karamu was intermediate in its susceptibility to FHB. Progeny of a cross between Karamu and Otane were selected for the remainder of this study because a doubled haploid population was available, enabling replication and cross-year comparisons of individual lines. For this reason, doubled haploid populations are particularly useful for investigating quantitative characters, such as FHB resistance.

Generation of epidemics of FHB in field trials can be difficult because of the requirement for both a source of nearby inoculum and high humidity during and preferably after anthesis. In New Zealand, FHB is most severe in wheat crops that follow maize, particularly when there is rainfall during anthesis and early grain fill (Cromey et al. 2002). Artificial inoculation and misting were used in this series of trials to maximise the likelihood of sufficient levels of FHB to compare cultivars and lines. This was successful in 2001/02 (mean FHB incidence 12\%), but only partly successful in 2002/03 (mean FHB incidence 2\%).

Measurements of visibly infected grains were not well correlated between years, or with other measurements in either year. Measuring visibly infected grains is, therefore, not a good method to assess cultivar resistance to FHB. However, because this can be carried out relatively quickly on samples of harvested grain, this method may be useful for identifying highly susceptible lines, especially if carried out on samples from more than one trial.

While visible FHB levels were very low in 2002/03, the proportion of harvested grains with Fusarium was almost half that recorded in 2001/02 and allowed reasonable discrimination between lines. The correlation amongst lines between years for Fusarium incidence in harvested grain was high, which suggests it may be the most suitable measure of cultivar resistance under conditions of low visible disease.

In both years, there was a relationship amongst lines between Fusarium and mycotoxin levels in harvested grain samples. Many factors in addition to cultivar resistance influence actual mycotoxin accumulation (Cooney et al. 2001). Most of these, such as sources of inoculum, Fusarium species present and climate, will provide equal pressure across all lines in a trial. For this reason, correlations between Fusarium levels in grain and mycotoxin accumulation tend to be much higher within crops or trials than between sites (Cromey et al. 2002).

In both years, there was a relationship amongst lines between FHB incidence on ears and both Fusarium and mycotoxin levels in harvested grain. Each component contributes primarily to one major consequence of FHB. Visible FHB infection will have a direct effect on yield, as well as indicating the likely build-up of Fusarium and mycotoxins in grains. Fusarium infection in grains will affect seed germination and seedling vigour as well as enable mycotoxin accumulation to occur, while mycotoxins affect the end-use quality of grain harvested from a crop. However, since correlations between measurements were not always good, measurements of all of these three FHB components is likely to give the most accurate measure of FHB resistance in breeding lines or cultivars.

\section{ACKNOWLEDGEMENTS}

John Koolaard provided valuable comments on describing relationships between FHB components. This work was funded by the New Zealand Foundation for Research, Science and Technology. 


\section{REFERENCES}

Bai, G.; Shaner, G. 1994: Scab of wheat: prospects for control. Plant Disease 78: 760766.

Burgess, L.W.; Summerell, B.A.; Bullock, S.; Gott, K.P.; Backhouse, D. 1994: Laboratory manual for Fusarium research. $3^{\text {rd }}$ edition. University of Sydney, Sydney. 133 p.

Cooney, J.M.; Lauren, D.R.; di Menna, M. 2001: Impact of competitive fungi on trichothecene production by Fusarium graminearum. J. Agric. Food Chem. 49: 522-526.

Cromey, M.G.; Lauren, D.R.; Parkes, R.A.; Sinclair, K.I.; Shorter, S.C.; Wallace, A.R. 2001a: Control of Fusarium head blight of wheat with fungicides. Aust. Plant Path. 30: 301-308.

Cromey, M.G.; Parkes, R.A.; Fraser, P.M. 2001b: Fusarium levels in grain harvested from New Zealand wheat and barley crops in 2000. N.Z. Plant Prot. 54: 193-197.

Cromey, M.G.; Shorter, S.C.; Lauren, D.R.; Sinclair, K.I. 2002: Cultivar and crop management influences on Fusarium Head Blight and mycotoxins in spring wheat (Triticum aestivum) in New Zealand. N.Z. J Crop Hort. Sci. 30: 235-247.

Dill-Macky, R.; Jones, R.K. 2000: The effect of previous crop residues and tillage on Fusarium head blight of wheat. Plant Disease 84: 71-76.

Lauren, D.R.; Agnew, M.P.; Smith, W.A.; Sayer, S.T. 1991: A survey of the natural occurrence of Fusarium mycotoxins in cereals grown in New Zealand in 19861989. Food Additives and Contaminants 8: 599-605.

Lauren, D.R.; Ringrose, M.A. 1997: Determination of the fate of three Fusarium mycotoxins through wet-milling of maize using an improved HPLC analytical technique. Food Additives and Contaminants 14: 435-443.

Parry, D.W.; Jenkinson, P.; McLeod, L. 1995: Fusarium ear blight (scab) in small grain cereals - a review. Plant Path. 44: 207-238.

Sayer, S.T.; Lauren, D.R. 1991: Fusarium infection in New Zealand grain. N.Z. J. Crop Hort. Sci. 19: 143-148.

Snijders, C.H.A. 1990: Genetic variation for resistance to Fusarium head blight in bread wheat. Euphytica 50: 171-179. 